\title{
In Situ Study of High-Temperature Mechanical Properties of Carbon Nanotube Scaffolds
}

\author{
Sanjit Bhowmick ${ }^{1}$, Chandra Sekhar Tiwary ${ }^{2}$, S. A. Syed Asif ${ }^{1}$ and Pulickel M. Ajayan ${ }^{2}$ \\ 1. Bruker Corporation, Minneapolis, USA \\ 2. Department of Material Science and Nano Engineering, Rice University, Houston, USA
}

Arrays of vertically aligned carbon nanotubes (CNTs), also known as forests, turfs or scaffolds, have received significant attention recently for potential use in applications such as thermal, electrical, interface materials, interconnects, energy absorbing foams, biologically inspired adhesives, filtration structures, and composite reinforcement agents [1-4]. In this work, blocks of aligned CNTs were grown by chemical vapor deposition using ferrocene and xylene on silicon wafer substrates. Prior to the growth process, the surface of the substrate was sputter by $10 \mathrm{~nm}$ aluminum and $1.5 \mathrm{~nm}$ iron films. The density of the $3 \mathrm{D}$ structure was measured to be $0.13-0.32 \mathrm{mg} / \mathrm{mm}^{3}$. The nucleation source agent, time, and temperature generally determine the dimension, morphology, density, and tortuosity of the scaffold. To conduct in situ pillar compression tests, micropillars of dimensions $15 \mu \mathrm{m}$ x $15 \mu \mathrm{m}$ in cross-section and 25-30 $\mu \mathrm{m}$ in height were prepared from the middle of the sample by focused ion beam (FIB).

Quasistatic and dynamic uniaxial compression experiments were conducted with a $20 \mu \mathrm{m}$ flat punch diamond probe attached to an in situ nanomechanical instrument, PI 88 SEM PicoIndenter. Using the displacement-controlled feedback, the pillars were compressed to 20-30\% strain at a strain rate of $10^{-3} \mathrm{~s}^{-}$ ${ }^{1}$ at room temperature (RT) as well as several elevated temperatures up to $800^{\circ} \mathrm{C}$. Heating was achieved through closed-loop resistive heating of both the probe and sample. In situ mechanical testing allows precise alignment of the tip with the sample as well as direct and real-time observation of the deformation process.

The microstructure of the sample and cross-section are shown in fig. 1. Fig. 2a shows a pillar sample milled by FIB. Fig. 2b displays stress-strain curves which are calculated from load-displacement data obtained from two tests conducted at room temperature and at $700^{\circ} \mathrm{C}$. The plot indicates that stress increases linearly with strain until a critical stress (A) at which stress dropped to a lower stress plateau, called instability stress (B). As the strain on the structure increases, several stress drops can also be observed. Microstructural observation during compression of the pillars confirms that critical stress (A) is associated with the formation of the first buckling wrinkle at the bottom of the pillars. With increasing strain, additional wrinkles are formed. Fig $2 \mathrm{~b}$ also indicates that elastic moduli of the structure remain nearly constant at room temperature and $700^{\circ} \mathrm{C}$ whereas critical stress for first buckling formation decreases by $46 \%$ at $700^{\circ} \mathrm{C}$ compared to RT. The stress drop associated with first buckle formation in the sample is much higher at RT compared to elevated temperature. The small stress drops after the initial instability (B) are the result of the formation of other buckling wrinkles. The SEM image in fig. 3 shows low and high magnification of the deformation zone at the base of a pillar. The formation of buckling at the bottom layer of the CNT pillars is a dominating deformation mechanism which makes this structure unique compared to other similar porous and low density 3D materials.

\section{References:}

[1] S Ozden et al, Adv. Mater. 27 (2015), p. 1842.

[2] SD Mesarovic et al, Scripta Mater. 56 (2007), p. 157. 
[3] AA Zbib et al, Nanotechnology 19 (2008), p. 175704.

[4] SB Hutchens, LJ Hall and JR Greer, Adv. Funct. Mater. 20 (2010), p. 1.
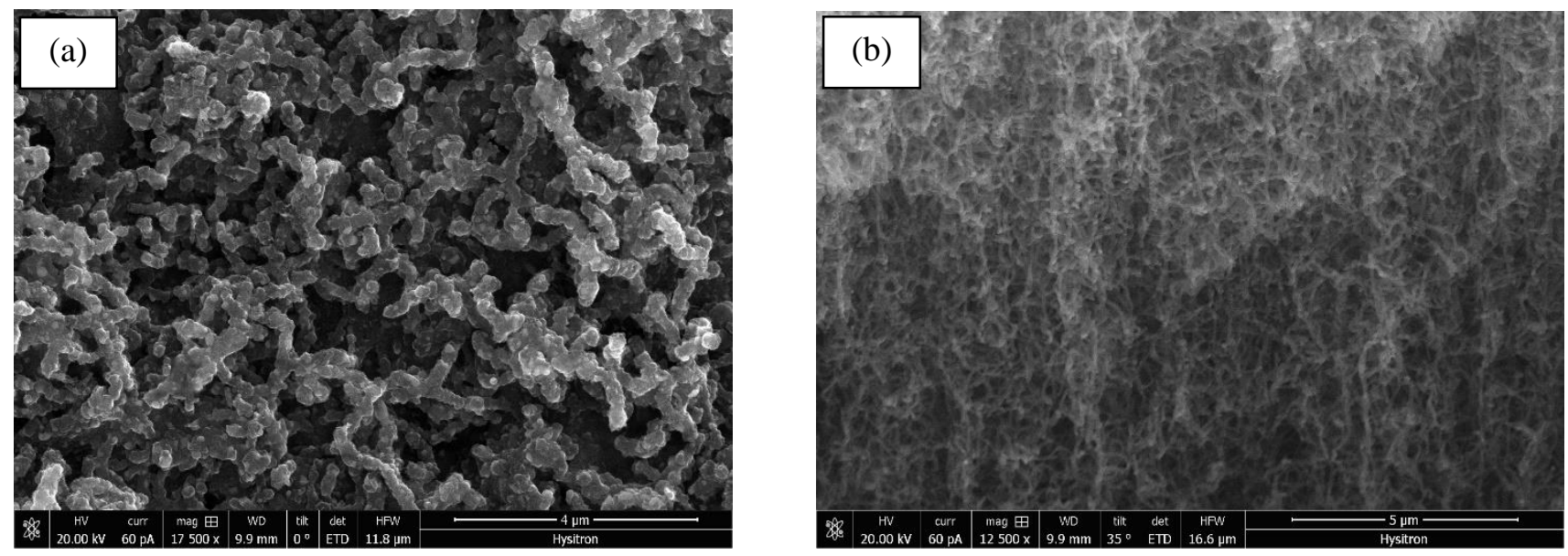

Figure 1. Microstructure of CNT scaffold: (a) surface of the sample (b) cross-section
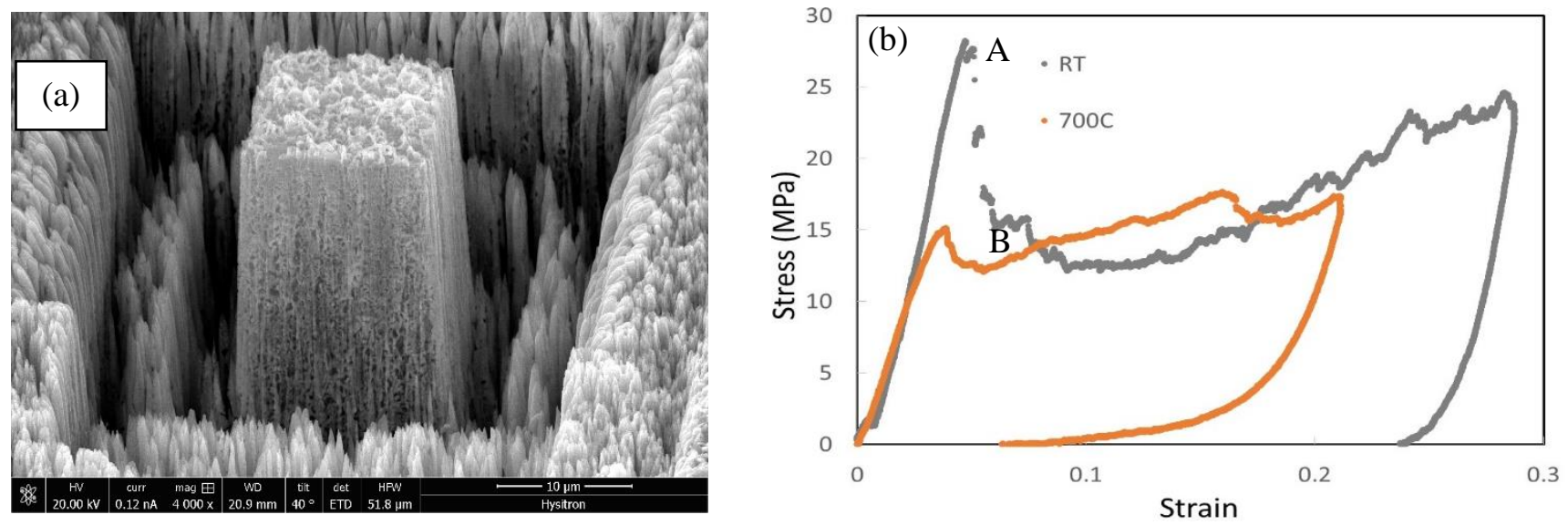

Figure 2. (a) A Fib-milled pillar (b) stress-strain curves obtained at RT and $700^{\circ} \mathrm{C}$.
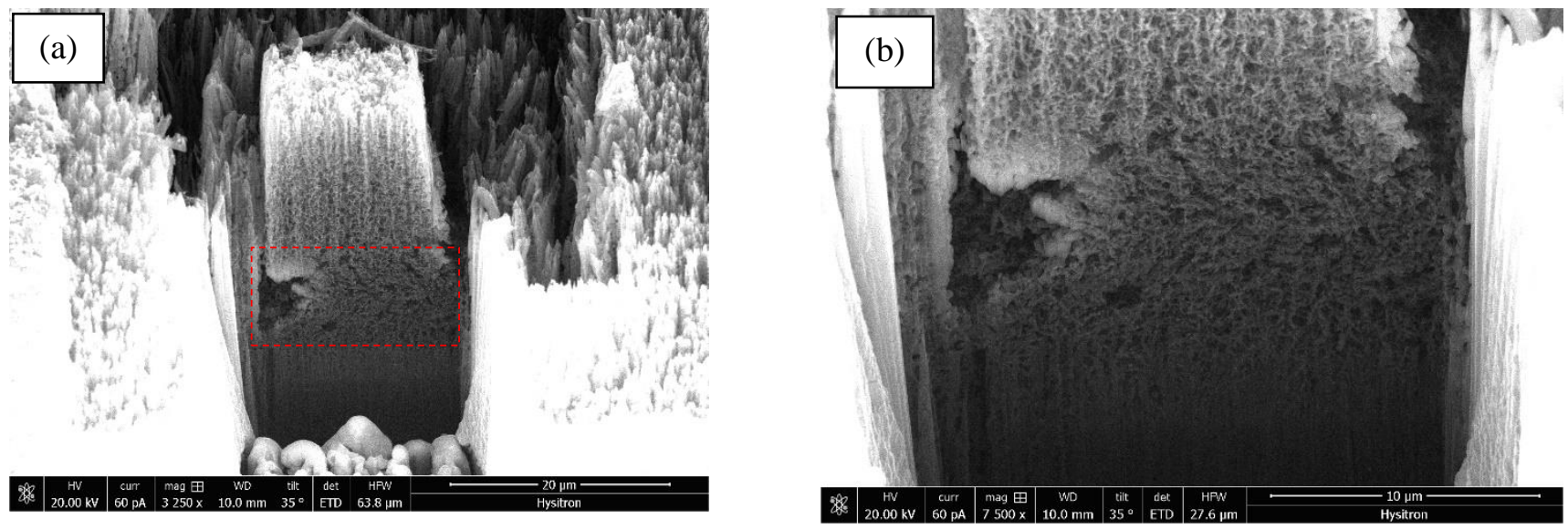

Figure 3. (a) low magnification and (b) high magnification images showing deformation at the base of the pillars. 